\title{
The Green Biofuel Conundrum: Technological Advances Versus Economic
} and Environmental Implications

\section{Shivasuriya Sanath*, Shaposhnikov Roman, Sola Lorenzo and Sperindio Riccardo}

Department of Biology and Biotechnology "Lazzaro Spallanzani", University of Pavia, Italy

*Corresponding Author: Shivasuriya Sanath, Department of Biology and Biotechnology "Lazzaro Spallanzani", University of Pavia, Italy.

ORCID ID: https://orcid.org/0000-0003-2497-9279
Received: April 15, 2021

Published: June 25, 2021

(C) All rights are reserved by Shivasuriya

Sanath., et al.

\section{Abstract}

Interest in biofuels production and usage has increased worldwide as part of government policies to address the growing scarcity and riskiness of petroleum use, and, at least in theory, to help mitigate adverse global climate change caused by them. In this written assay the authors have dealt some different methods to produce biofuels and their environmental and economic impact. They focused on oleaginous microorganisms, organisms that can accumulate a great amount of lipids; belong to this class some yeasts, fungi, bacterium and microalgae. The latters are microscopic photosynthetic microorganisms that represent the most important oleaginous microorganisms in terms of production. Indeed, another topic treated in this work is the microalgae genetic engineering modifications, such as overexpression or downregulation of genes involved in the lipids metabolism, in order to produce a high amount of these molecules for the conversion of them into biodiesel. Subsequently, the beneficial effects on the environment derived from the combustion of this biofuel compared with its counterpart, the petrol diesel, have been described. The problems related to the cultivation on large-scale and the poor knowledge of basic biology that make impossible the industrial production of microalgae, have also been analyzed. On the other hand, another treated subject is the fungal and bacterial depolymerization of lignin in order to produce biofuels. The lignin, a plant derived compound, represent one of the most abundant polymers in the biosphere, unfortunately it presents a very complex structure with some chemically inert bond. For these reasons research is still at the beginning, so nowadays we cannot speak about polluting effects and large-scale production, with the consequent economic impact, of biofuel derived from this source.

Keywords: Biofuels; Microalgae; Biology

\section{Graphical Abstract}

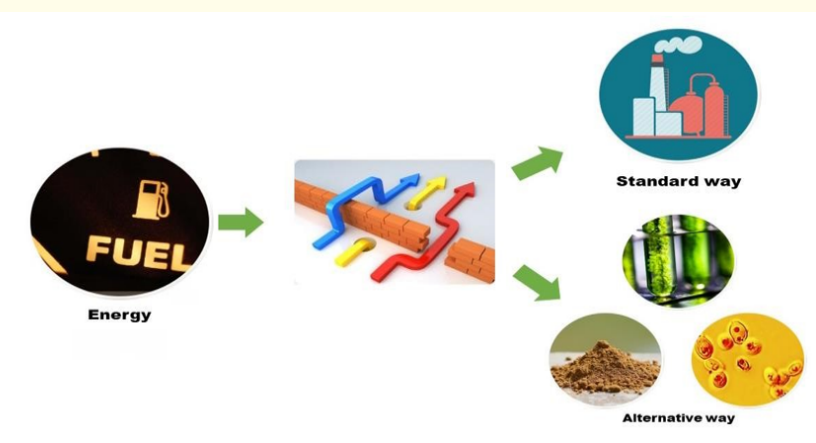

Figure a

\section{Biofuel production from oleaginous microorganisms}

Biodiesel, a mixture of fatty acid methyl esters (FAMEs) derived from animal fats or vegetable oils, is rapidly moving towards the mainstream as an alternative source of energy. However, biodiesel derived from conventional petrol or from oilseeds or animal fat cannot meet realistic need, and can only be used for a small fraction of existing demand for transport fuels. Therefore, oleaginous microorganism s are available for substituting conventional oil in biodiesel production. Most of the oleaginous microorganisms like microalgae, bacillus, fungi and yeast are all available for biodiesel production. Regulation mechanism of oil accumulation in microorganism and approach of making microbial diesel economically competitive with petrodiesel are discussed. 
One of the most prominent renewable energy resources is biodiesel, which is produced from renewable biomasss by transesterification of triacyl-glycerols, yielding monoalkyl esters of long-chain fatty acids with short-chain alcohols, for example, fatty acid methyl esters (FAMEs) and fatty acid ethyl esters (FAEEs). It contributes no net carbon dioxide or sulfur to the atmosphere and emits less gaseous pollutants than normal diesel. In the production of worldwide biodiesel, various renewable lipids have been chosen, including vegetable oils, animal fats and wasting oils. In South East Asia, Europe, United States and China, palm oil, rapeseed oil, transgenetic soybeans and wasting oil were used to produce biodiesel, respectively. However, all these plant oil materials require energy and acreage for sufficient production of oilseed crops [1].

To be a viable substitute for a fossil fuel, an alternative fuel should not only have superior environmental benefits over the fossil fuel it displaces, be economically competitive with it, and be producible in sufficient quantities to make a meaningful impact on energy demands, but also provide a net energy gain over the energy sources used to produce it. Oleaginous microorganisms are defined as microbial with the content of microbial lipid excess of $20 \%$. Biodiesel production using microbial lipids, which is named as single cell oils ( $\mathrm{SCO}$ ), has attracted great attention in the whole world. Although there are all kinds of microorganism storaging oils, such as microalgae, bacillus, fungi and yeast, not all of them are available for biodiesel production [28].

Numerous oleaginous yeasts and microalgaes have been reported to grow and accumulate significant amounts of lipids similar to vegetable oil, methyl-esters and soaps, used as sole carbon and energy sources. In recent years, a large number of hydrophobic lipid accumulating microorganisms have been studied as substrates for SCO production, especially used in the production of biodiesel. In microorganisms, the extent of lipid accumulation is determined by the genetic constitution, as maximum attainable lipid contents can vary enormously among species and even among individual strains. According to different microorganisms and different culture conditions (such as temperature, $\mathrm{pH}$, culture time, etc.), oil content and composition are different. Study showed that most microalgae biomasses are a rich source of $\mathrm{u} 3$ and $\mathrm{u} 6$ fatty acids, essential amino acids, like leucine, isoleucine, valine, etc. The accumulated oil in almost all microalgaes is mainly triglyceride (> 80\%), with a fatty acid profile rich in C16 and C18, showing 09, 012 and 015 desaturation, comparable with plant seed oil. Microalgae grow extremely rapidly and many are exceedingly rich in oil, and double their biomass within $24 \mathrm{~h}$. The average lipid content of algal cells varies between $1 \%$ and $70 \%$, but can reach $90 \%$ of dry weight under certain conditions. By now, the microalgal biomass market produces about $5000 \mathrm{t}$ of dry mass/year and generates a turnover of approximately
US $\$ 1.25 \times 10^{9} /$ year [24].

It has been believed as one of good candidates for biodiesel production, based on higher photosynthetic efficiency, higher biomass production and faster growth compared to other energy crops. Although microalgae are high lipid microbial, they need a larger acreages to culture algaes and long fermentation period than bacteria.

\section{Screening for potential oleaginous microorganism}

Although several wild-type oleaginous microorganisms are able to synthesize rich oil, these strains have a limited ability to produce biomass. Making use of mutation techniques in microbial lipid production to filtrate better strain will get much more biomass than wild-type. Recently, the Greece researcher reported that Mortierella isabellina cultivated in nitrogen-limited media presented remarkable cell growth (up to $35.9 \mathrm{~g} / \mathrm{L}$ ) and high glucose uptake even with initial sugar concentrations at $100 \mathrm{~g} / \mathrm{L}$ in media [22].

\section{Genetic and metabolic engineering}

With the development of biochemistry and molecular biology (such as DNA recombination, site directed mutagenesis), It is likely to have the greatest impact on improving the economics of production of microalgal diesel. Change in the degree of fatty acid unsaturation and decrease or increase of the chain length of fatty acids are the major challenges in modifying the lipid composition. All these are regulated by enzymes, but most of the enzymes are membrane bound, which has significantly hindered efforts to purify and study their function. Three most important interdependent genetic technologies are involved, including cloning genes of critical enzymes, transgenic expression of these genes aimed to achieve a fine highproduct microbial oil recombination strain, and modification of cloned genes in order to engineer the expressed protein. It has been mainly advanced in plants using DNA recombination [16].

\section{Making full use of by-products}

In addition to oils, oleaginous microorganisms contain significant quantities of proteins, carbohydrates and other nutrient contents. Therefore, how to make use of these by-products and improve their industrial value is another way to reduce the production cost in biodiesel. For example, the residual biomass from biodiesel production can be used potentially as animal feed, and also to produce methane by anaerobic digestion. The waste glycerol from the biodiesel industry could therefore be easily transformed into a precious chemical. Moreover, the price of the final product could be reduced considerably in this way [8].

\section{Microalgae 'omics' era for biofuels production}

Microalgae are microscopic prokaryotes or eukaryotes photosynthetic microorganisms that constitute the basis of aquatic food 
chains. Different phyla and classes belong to this phylogenetically multicolored group. The raising interest in the algal field is related to their versatile utilization. They are able to fix solar energy into biomass, they do not require freshwater, they can accumulate metabolites like carbohydrates under the form of starch and lipids in the form of triacylglicerol (TAG). Moreover they are characterized by a fast turnover rate representing an alternative to land crops and some microalgae are also able to produce high value-added products with medical, nutraceutical and pharmaceutical importance such as proteins, antioxidants and pigments. One of the hottest field is the microalgae-based production of biofuels, indeed, they can be used to obtain several types of biofuels like biodiesel, biomethane and bioethanol $[6,7,26,30]$.

The 'omics' era, accompanied by genome sequencing techniques and genetic engineering tools, contribute to discovery the pathways beside a variety of metabolic processes understanding the different gene and protein networks. In particular, electroporation, biolistic microparticle bombardment and Agrobacterium tumefaciens-mediated gene transfer have been used to deliver DNA into microalgal cells, RNA interference (RNAi) allows the repression or activation of genetic expression, while, genome editing techniques based on ZFNs, TALENs and CRISPR/Cas9 provide valuable tools to modify the gene sequences. The microalgae-based biofuel production mainly depends on lipid biosynthesis and on the reduction of lipogenesis-competitive pathways. In this perspective, in a recent review Chen and collaborators, after the analysis of 7170 papers related to microalgae, showed that, the five algae genera more studied for biofuel production are Chlamydomonas, Chlorella, Synechocystis, Phaedoactylum and Nannochloropsis [6,7,26,30]. Lipid production is characterized by three main steps called, malonylCoA synthesis, acyl chain elongation and triacylglycerol formation (TAG). There are also competitive pathways involved in lipid catabolism like $\beta$-oxidation [21]. An overview of the microalgal lipid biosynthesis is shown in figure 1 [14]. Early efforts were focused in increasing the expression of enzymes involved in the fatty acid synthesis like the acetyl-CoA carboxylase (ACCase) which catalyzes the carboxylation of acetyl-CoA to form malonyl-CoA with limited success. Instead, interesting results were obtained acting on genes involved in TAG formation, the final step of lipid production. One striking example is represented by the overexpression of diacylglycerol acyltransferase (DGAT) gene that results in TAG accumulation. Two types of DGAT are involved in TAG formation in microalgae, one located in the chloroplast, DGAT1, and the other one in the ER membrane, DGAT2. In particular, the overexpression of DGAT2 from Chlamydomonas reinhardtii in Scenedesmus Obliquus leads to an increase in TAG production $[6,7,26,30]$. An alternative strategy is to decrease the lipid catabolism and one favourite tar- get is Boxidation, the most competitive pathway to lipogenesis. $\beta$-oxidation breaks down fatty acids in cytosol or mitochondria and peroxisome in prokaryotes and eukaryotes respectively. Knockingdown or inhibiting the $\beta$-oxidation pathway lead to an increase in lipid production [25]. Another reasonable approach is to increase the quality of lipids acting on fatty acyl-ACP thioesterase (FAT) to produce ideal fatty acids with a chain length of 12 and 14 [25]. In many species stressful conditions like salinity, $\mathrm{pH}$, temperature and nutrient starvation ( $\mathrm{N}$ and $\mathrm{P}$ ) induce accumulation of storage metabolites like lipids and carbohydrates. Recent studies using a omic approach identified transcriptional factors involved in lipid metabolism, possibly in the TAG biosynthesis. One of these is PSR1 from Chlamydomonas reinhardtii, a key regulator of nutrient starvation-induced TAG biosynthesis [3]. Another TF involved in TAG biosynthesis under nutrient starvation is CHT7 from Chlamydomonas, mutating the latter it is possible to obtain TAG accumulation without affecting biomass [32].

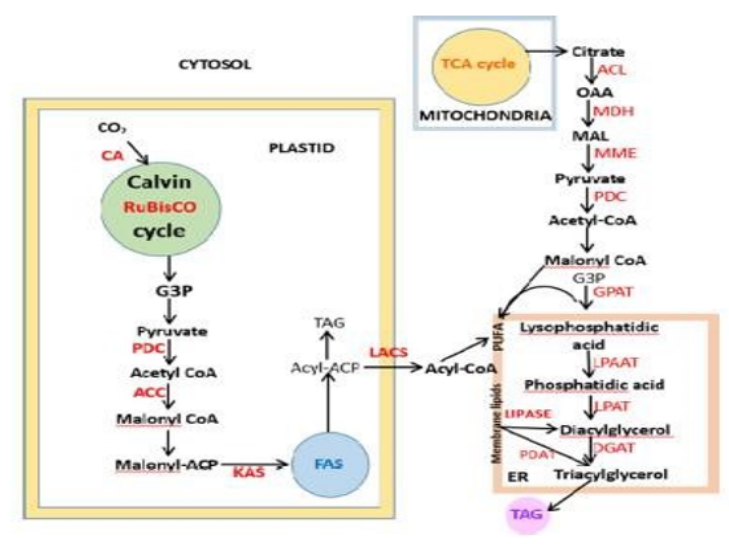

Figure 1: Scheme representing the pathways involved in microalgal lipid byosinthesis (14. Jagadevan et al. 2018).

Others interesting approaches are based on the manipulation of genes that are not directly involved in the lipid metabolism. In this light the main target is the ribulose-1,5-bisphosphate carboxylase/oxygenase (Rubisco) essential for biomass productivity which in turn determines the rate of oil productivity. This enzyme is responsible for the first step of carbon fixation during the Calvin cycle i.e. the carboxylation of ribulose 1,5-bisphosphate (RuBP) in 3-phosphoglycerate. It is also involved in a side reaction in which RuBP and oxygen are converted into 2-phosphoglycolate, a toxic compound recycled by the photorespiratory pathway. Different research in order to enhance the Rubisco selectivity toward car- 
bon fixation have had limited success due to complex kinetics of this protein. More promising studies in Nannochloropsis oceanica have shown that targeting factors involved in the regulation of the Rubisco activity, such as the Rubisco activase (RCA) enzyme, a protein that uses the hydrolysis of ATP for regenerating the Rubisco catalytic site, resulted in elevated photosynthetic activity and lipid accumulation [35]. However, other key enzymes entailed in the Calvin cycle such as fructose 1,6-bisphosphate aldolase (aldolase) and 1,7bisphosphatase (SBPase) are supposed to increase the biomass yield. In addition, it has been reported in Phaeodactylum tricornutum that preventing the expression of the pyruvate carboxylase kinase (PDK) results in an increase of total lipid content of algal cells [19]. Indeed, PKD inactivates through phosphorylation the mytochondrial pyruvate dehydrogenase complex (PDC) that is responsible for the conversion of pyruvate to acetyl-CoA, the substrate of ACCase. So, inhibhition of PDK prevents the phosphorylation of the PDC, which in turn, determines an enhanced production of acetyl-CoA. The latter following subsequently transformations will contribute to TAG formation. Moreover, Deng and collaborators demonstrated that the repression by RNAi of the CrCO gene in Chlamydomonas reinhardtii, a homologous gene of the circadian-regulated CONSTANS gene (CO) that plays an important role in photoperiod and flowering time, leads to a boost in lipid and TAGs levels [11].

Nowadays the explosion of 'omics' techniques including genomics, proteomics, metabolomics and the combination between them represent a great achievement in the comprehensive understanding of all the complex pathways beside apparently simply organisms such as microalgae. However, our vision in microalgal basic biology is still poor. In addition genetic engineering methods have some limitations mainly linked to low transformation success, high production cost and low growth rate. Moreover GM microalgae, such as GM plants and other GM organisms, must be carefully analyzed and accepted before being released in the environment and utilised in industrial process. The microalgal field, anyway, represents one of the more promising field toward biofuel production.

\section{Lignin valorisation as a potential source of biofuel}

Lignin can be potentially one of the most abundant sources of aromatic compounds on our planet. However, until several years ago it was considered practically a waist, because of its intrinsic recalcitrance. Lignin plays an important role as a structural polymer of the cell wall of vascular plants and several algae. From chemical point of view, it is an organic polymer highly rich with phenolic groups, it is particularly rich with following three: guaiacyl (G), syringyl ( $\mathrm{S}$ ), and hydroxyphenyl $(\mathrm{H})$ cross linked by enzymatic catalysis $[27,29]$. Lignin can be especially important as an alternative, re- newable energy resource [33]. Potential high-value products from isolated lignin include low-cost carbon fiber, engineering plastics and thermoplastic elastomers, polymeric foams and membranes, and a variety of fuels and chemicals all currently sourced from petroleum [26]. Particularly for a jet fuel around 15-20\% of aromatic compounds has to be present in mixture, and lignin seem to be an excellent potential source of such compounds [12]. Search of new lignin depolymerization methods economically is highly proficient as without new applications total mass of lignin produced will relatively quickly exceed the current world market necessity in lignin [31] especially if we take in consideration that production of lignin can actually grow up with wider production of cellulosic bioethanol. Some research show that US bioethanol industry alone will generate up to 60 Mtonne/yr lignin by 2022 [13].

Valorization of lignin is a hard challenge as there are several serious problems. First, the chemical bond type is tough for enzymatic cleavage as it is composed of $\mathrm{C}-\mathrm{O}$ or $\mathrm{C}-\mathrm{C}$ bonds, that are chemically inert. That is why it is necessary applicate some non-mainstream biotechnological and bioengineering techniques to obtain an optimal for further processing type of lignin. Second, natural lignin and natural-like lignin obtained by some industrial processes are not only chemically inert, but also non soluble in water, thus it is even more important to choose wisely which technological process should be applied for industrial outcome optimization. Third lignin chemical structure do not have an ordinal pattern. This is a big challenge for enzymatic catalysis as it is usually highly specific it its substrate [4].

It is also important to remember that there are some upstream methods of optimization of final lignin product. For instance, until now most bioengineering methods was applied to decrease the lignin content, but as we said before lignin can potentially be one of the largest renewable sources of essential for humanity chemical substances. Rinaldi., et al. [29] in their work suggest utilizing bioengineering methods to come up with better natural lignin structure directly from cultivated plants. Even if this is not a trivial task due to the time needed to pass from plants utilized nowadays to new type genetically modified ones, it will certainly bring profit. Especially it is important when we talk about softwood and hardwood as the time in this fields of industry can be expanded from 5 to 30 years [29]. As we already mentioned downstream lignin elaboration is a critical point, as it defines lignin availability to be elaborated into substrates with economic value. From chemical point of view lignin depolymerization can be done via several reaction types: pyrolysis, hydrogenolysis, oxidation, gasification and combustion. Each of these pathways leads to different final products described in figure 2 [34]. 
nolic aromatic targets [2]. Generally, bacteria that are capable to degrade lignin do not utilize lignolytic peroxidases as most fungal species that are utilized in similar biotechnological processes. In most cases bacterial strains utilize DyP-type peroxidases and laccases. It was demonstrated that bacterial laccases are less effective in comparison with fungal ones, however there are several advantages in their application, such as thermo tolerance and halotolerance. These two aspects can potentially be very important if we speak about biotechnological application [10]. There are some studies that showed a possibility to convert lignin depolymerization products into alcohols and phenols. Particularly, application of the high selective catalytic hydrogenation process with $\mathrm{Ni} / \mathrm{SiO} 2$, as well as several other Ni-based catalysts, show an excellent result in converting guaiacol to cyclohexanol. It is important to mention that such conversion process has low effect on catalyst, so it has high potential for industrial application [30,35,36].

Figure 2: Chemical pathway influence on the final lignin depolymerization product. (34. Vavilala et al. 2019).

\section{Conclusions}

Nowadays, the majority of the energy systems in the world are

Current necessity of chemical pretreatment of biomass that contain lignin is a huge negative aspect as the total outcome yield of depolymerization of such product is much lower in comparison with non-treated lignin [2]. That is why there is whole research sector which is occupied with search of alternatives for lignin-containing biomass pretreatment. Unfortunately, diversity of lignin chemical bonds has restricted natural organisms capable to degrade lignin to several fungal and bacterial species. Fungal lignin degradation has been studied the most and it was shown that white-rot fungi are one of the most effective fungal classes in lignin depolymerization [18]. There are only few wild type bacterial strains that are capable of lignin degradation. In general, Sterptomyces, Rhodococcus, Pseudomonas, and Bacillus strains have been reported to have lignin decomposition ability [17]. Main enzymatic groups utilized by these species are peroxidases and laccases. Peroxidases capable to depolymerize lignin share some structural similarities. In particular they have an increased redox potential and show specificity to non-phenolic aromatic groups or $\mathrm{Mn}^{2+}$ in LiP or MnP respectively [20]. One of the most utilized peroxidase subfamily is DyP-type peroxidases with can be divided in four major subclasses (A-D) where $\mathrm{A}-\mathrm{C}$ are primely found in bacteria and $\mathrm{D}$ subclass is found in fungal species [37].

It was demonstrated that type A DyP-type peroxidases have Tatdependent secretion signal. This knowledge permits to suggest, that type A DyP-type peroxidases can be involved into extracellular lignin depolymerization $[9,10]$. Second enzymatic group, laccases, has slightly different properties. They are able to oxidize aromatic phenols and amines, and in some cases can even oxidize non-phebased on fossil fuels, but unfortunately there are some important issues related to the use of these latters, in particular, negative environmental impacts, such as global warming, and pollution. Another problem of the fossil fuels is the existence of limited reserves, that contribute to make it a non-renewable energy resource. It is established nowadays that current energy consumption derived from fossil fuels and their production patterns are unsustainable in the long term, and this is due to their exhaustion and consequent price increase. Thus, current and future energy sources should be more sustainable, for instance, it is necessary that they are renewable and cause lower negative impacts from an ecological and economical point of view [5].

At this point a question arises: can biofuels produced with the methodologies described by the authors before be a good sustainable source of renewable energy? To answer at this question, we need to consider some important aspects. Previously we have seen the methods to increase the production of lipids in oleaginous microorganism in order to use them for the production of biodiesel. The biodiesel, a well-known biofuel, from an environmental point of view result less pollutant than its counterpart, the petroleum derived diesel. Indeed, the quantity of carbon dioxide, polycyclic aromatic hydrocarbons, carbon monoxide and particulate matters released during the combustion process of biodiesel is lower than those emitted by petrol diesel [38]. Then, in order to be competitive with the production of fossil fuels and its large-scale production is required. Today one of the main studied method to obtain from oleaginous microorganism a large amount of lipids for the conversion of them in biofuels is the microalgal cultivation, and there are two ways to achieve this: the pond systems and the 
photobioreactors (PBRs). The latters are closed and automated systems that control variables, including nutrients, $\mathrm{pH}$, light, and temperature when cultivating microalgae at large scale. Unfortunately, because of their automation, they have a very high price. On the other hand, the pond systems, an opened outdoor cultivation tank, have the advantage of having a lower price than the PBRs but this cultivation method not provide control over other organisms that can contaminate the pond. Another very important disadvantage of this systems is the impossibility to use genetically modified microalgae because these organisms are not allowed by policymakers as a result for possible contamination risk of the surrounding environment []15. Moreover, according to Chen and collaborators despite screening more than 10,000 microalgal strains, not a single alga has passed the large-scale commercial manufacturing for biofuel production, indeed advancements in applied technologies for microalgal biofuel production depend on the progress of basic biology; our current limited understanding of microalgae biology remains the biggest obstacle towards the industrialization of microalgal biofuel [6]. For what concerns biofuel derived from lignin we have to say that the conversion of this polymer moieties into biofuels and other high valueadded products, as already said previously, are still challenging to the researchers due to the heterogeneity, complex and inert structure of lignin [23]. For these reasons the research in this field is still at the beginning so we cannot yet speak about large-scale production and potential pollution of these biofuels. Now, having this information regarding the pain and gain of the biofuels produced with the methodologies treated before, we can establish if they are a good sustainable renewable source. Unfortunately, there are still many issues, in particular the costs and the lack of knowledge which currently don't allow to be competitive with the fossil fuels. Even if nowadays, there are these important problems, in the future surely, thanks to the technology and research improvement these biofuels will play a key role in our energetic production.

Writing our paper we understood the importance of collaboration during daily life but this experience also give us the opportunity to realize how important is working together expecially in the scientific community. Of course it is not an easy task because working like a team means be comprehensive learning to mix different ideas and adding creativity to reach the best result as possible. This year our work has been made more difficult because of the COVID-19 pandemic not allowing to meet together phisically. Anyway, in conclusion, we are satisfied about our work and we look forward to have the feedback by our referees.

\section{Authors' Contribution}

The essay presented is the accomplishment of the cooperation among all the authors, providing a general overview of the topic, aiming to create the most accurate vision possible. Precisely, Shivasuriya S. examined biodiesel production starting from oleaginous microorganisms. Shaposhnikov R. engaged with lignin utilisation as an analogue to currently known energy sources, concentrating on its biochemical features and he also created the graphical abstract. Sola L. studied the relationship between microalgae and biofuel production, focusing on the lipid metabolism and he managed the references. Sperindio R. merged all the different themes writing the abstract, the conclusions and he also responsible for the entire paper layout. All members of the group participate actively to the realization of the article expressing their perspective and revisioning the entire work.

\section{Bibliography}

1. Antolin G., et al. "Optimisation of biodiesel production by sunflower oil transesterification". Bioresource Technology 83 (2002): 111-114.

2. B Fisher., et al. "Lignin biodegradation and industrial implications". AIMS Bioengineering 1.2 (2014): 92-112.

3. Bajhaiya A K., et al. "PSR1 is a global transcriptional regulator of phosphorus deficiency responses and carbon storage metabolism in Chlamydomonas reinhardtii". Plant Physiology 170.3 (2016): 1216-1234

4. Bugg TD H and Rahmanpour R. "Enzymatic conversion of lignin into renewable chemicals". Current Opinion in Chemical Biology 29 (2015): 10-17.

5. Caetano N S., et al. "New Trends in Energy Production and Utilization”. Energy Procedia 107 (2017): 7-14.

6. Chen C., et al. "Expression of type 2 diacylglycerol acyltransferse gene DGTT1 from Chlamydomonas reinhardtii enhances lipid production in Scenedesmus obliquus". Biotechnology Journal 1 (2016): 336-344.

7. Chen H., et al. "Ten years of algal biofuel and bioproducts: gains and pains". Planta 249.1 (2019): 195-219.

8. Chisti Y. "Biodiesel from microalgae". Biotechnology Advances 25.3 (2007): 294-306.

9. Colpa D I., et al. "DyP-type peroxidases: A promising and versatile class of enzymes". Journal of Industrial Microbiology and Biotechnology 41.1 (2014): 1-7.

10. de Gonzalo G., et al. "Bacterial enzymes involved in lignin degradation”. Journal of Biotechnology 236 (2016): 110-119. 
11. Deng X., et al. "A photoperiod-regulating gene CONSTANS is correlated to lipid biosynthesis in chlamydomonas reinhardtii". BioMed Research International (2015).

12. Hileman J I., et al. "Energy Content and Alternative Jet Fuel Viability". Journal of Propulsion and Power 26.6 (2010): 11841196.

13. Holladay J E., et al. "Top Value-Added Chemicals from Biomass Volume II - Results of Screening for Potential Candidates from Biorefinery Lignin". Prepared for the U.S. Department of Energy under Contract DE-AC05-76RL01830. II (October) (2007).

14. Jagadevan S., et al. "Recent developments in synthetic biology and metabolic engineering in microalgae towards biofuel production". Biotechnology for Biofuels 11.1 (2018): 1-21.

15. Johnson T J., et al. "Photobioreactor cultivation strategies for microalgae and cyanobacteria. Biotechnology Progress 34.4 (2018): 811-827.

16. Kalscheuer R., et al. "Microdiesel: Escherichia coli engineered for fuel production". Microbiology 152.9 (2006): 2529-2536.

17. Lee S., et al. "Bacterial Valorization of Lignin: Strains, Enzymes, Conversion Pathways, Biosensors, and Perspectives". Frontiers in Bioengineering and Biotechnology 7 (2019): 1-18.

18. Leonowicz A., et al. "Biodegradation of lignin by white rot fungi”. Fungal Genetics and Biology 27.2-3 (1999): 175-185

19. Ma YH., et al. "Antisense knockdown of pyruvate dehydrogenase kinase promotes the neutral lipid accumulation in the diatom Phaeodactylum tricornutum". Microbial Cell Factories 13.1 (2014): 1-9.

20. Martínez Á T., et al. "Biodegradation of lignocellulosics: Microbial, chemical, and enzymatic aspects of the fungal attack of lignin". International Microbiology 8.3 (2005): 195-204.

21. Ng I S., et al. "Recent Developments on Genetic Engineering of Microalgae for Biofuels and Bio-Based Chemicals". Biotechnology Journal 12.10 (2017): 1-13.

22. Papanikolaou S., et al. "Repression of reserve lipid turnover in Cunninghamella echinulata and Mortierella isabellina cultivated in multiple-limited media". Journal of Applied Microbiology 97.4 (2004): 867-875.

23. Ponnusamy V K., et al. "A review on lignin structure, pretreatments, fermentation reactions and biorefinery potential". Bioresource Technology 271 (2019): 462-472.
24. Pulz $\mathrm{O}$ and Gross W. "Valuable products from biotechnology of microalgae". Applied Microbiology and Biotechnology 65.6 (2004): 635-648

25. Radakovits R., et al. "Genetic engineering of algae for enhanced biofuel production". Eukaryotic Cell 9.4 (2010): 486-501.

26. Ragauskas A J., et al. "Lignin valorization: Improving lignin processing in the biorefinery". Science 344.6185 (2014).

27. Ralph J., et al. "Lignins: Natural polymers from oxidative coupling of 4-hydroxyphenyl- propanoids". Phytochemistry Reviews 3.1-2 (2004): 29-60.

28. Ratledge C. "Fatty acid biosynthesis in microorganisms being used for Single Cell Oil production:. Biochimie 86.11 (2004): 807-815.

29. Rinaldi R., et al. "Paving the Way for Lignin Valorisation: Recent Advances in Bioengineering, Biorefining and Catalysis". Angewandte Chemie - International Edition 55.29 (2016): 81648215.

30. Shu R., et al. "Hydrogenation of lignin-derived phenolic compounds over step by step precipitated Ni/SiO2". RSC Advances 6.7 (2016): 5214-5222.

31. Stewart D. "Lignin as a base material for materials applications: Chemistry, application and economics". Industrial Crops and Products 27.2 (2008): 202-207.

32. Tsai C H., et al. "The protein compromised hydrolysis of triacylglycerols 7 (cht7) acts as a repressor of cellular quiescence in Chlamydomonas". Proceedings of the National Academy of Sciences of the United States of America 111.44 (2014): 1583315838.

33. Tuck C 0. "Valorization of biomass: Deriving more value from waste (Science (695))". Science 338.6107 (2012): 604.

34. Vavilala S L., et al. "Lignin: Understanding and exploring its potential for biofuel production". In Advanced Bioprocessing for Alternative Fuels, Biobased Chemicals, and Bioproducts: Technologies and Approaches for Scale-Up and Commercialization. Elsevier Inc (2019).

35. Wei L., et al. "Enhancing photosynthetic biomass productivity of industrial oleaginous microalgae by overexpression of RuBisCO activase". Algal Research 27 (2017): 366-375.

36. Xu Y., et al. "In situ hydrogenation of phenolic compounds over Ni-based catalysts: Upgrading of lignin depolymerization products". New Journal of Chemistry 44.13 (2020): 5088-5096. 
37. Yoshida T and Sugano Y. "A structural and functional perspective of DyP-type peroxidase family". Archives of Biochemistry and Biophysics 574 (2015): 49-55.

38. Živković S and Veljković, M. "Environmental impacts the of production and use of biodiesel". Environmental Science and Pollution Research 25.1 (2018): 191-199.

Volume 4 Issue 7 July 2021

(C) All rights are reserved by Shivasuriya Sanath., et al. 